\title{
DIRECT NUMERICAL MODELLING OF FORMATION OF WELD METAL DENDRITE STRUCTURE WITH DISPERSE REFRACTORY INOCULANTS
}

\author{
D.Yu. ERMOLENKO, A.V. IGNATENKO and V.V. GOLOVKO
}

E.O. Paton Electric Welding Institute, NASU

11 Kazimir Malevich Str., 03680, Kiev, Ukraine. E-mail: office@paton.kiev.ua

\begin{abstract}
Studied were the advantages of alloy feeding with the disperse refractory inoculants for regulation and optimizing the primary structure of high-strength low-alloy steel weld metal. The main assumptions and limitations of a model of weld metal solidification process, proposed in this work, were considered. The model of melt solidification was described in details taking into account effect of the introduced disperse refractory inoculants. A hypothesis was presented on mechanism of interaction of a refractory inoculant with moving solidification front, which was taken in computations. Effect of the different alloying elements on surface energy of phase interface was described. Boundary conditions of the proposed model, realized in form of author software, were described. The results are given of computational experiments showing the possibilities of regulation of parameters and morphology of the primary structure of high-strength low-alloy steel weld metal by means of melt feeding with the disperse refractory inoculants (by example of $\mathrm{TiO}_{2}$ introduction). Presented is a comparison of experimental and computational results of change of the primary structure parameters. Developed mathematical model and software, written on its basis, are good for prediction of dimensional parameters and morphology of the weld metal primary structure considering refractory inoculants being fed in the weld pool. 17 Ref., 1 Table, 10 Figures.
\end{abstract}

Keywords : dendrite structure, disperse refractory inoculants, solidification, high-strength low-alloy steels, morphology, mathematical modelling

Continuous increase of the requirements to metal structure safety promotes for the necessity of mastering new steel grades with higher level of mechanical properties. In practice of metallurgical production a volume of high-strength low-alloy steels (HSLA) production continuously rises. The main tendencies in optimizing the properties of HSLA steels are reduction of content of alloying elements, increase of total number of microalloying elements, decrease of content of additives and residual elements promoting metal degradation, improvement of structural inhomogeneity, rise of mechanical properties level [1].

Alloy feeding with the refractory inoculants is a perspective method for optimizing the structure and properties of HSLA steels due to regulation, first of all, of structure parameters and, respectively, weld metal mechanical properties. It is known that grain size of the primary structure effects the nature of $\gamma \rightarrow \alpha$ transformations. If nucleation of $\alpha$-phase in the disperse dendrite structure starts at grain boundary in the upper area of bainite transformation, then nucleation of ferrite inside the primary grains at boundaries with non-metallic inclusions at temperatures close to bainite transformation end [2] is typical for coarser dendrites.
Work [3] considers a series of models of primary structure development and their critical analysis is carried out from point of view of possibility of their application for simulation of weld metal solidification process taking into account effect of the disperse refractory inoculants. Present work proposes a model based on finite element method coupled with cellular automation method.

Main assumptions and limitations of the model of metal solidification process. Studied system includes the refractory inoculants distributed in a liquid phase and significantly effecting solidification process.

Metal solidification process considering effect of the refractory inoculants can be considered as a result of interaction of two competing processes, namely high-rate movement of solidification front, caused by local non-equilibrium of diffusion processes at phase interface, and effect of the inoculants on local surface tension at phase interface, which has significant effect on formation of the weld metal primary structure of HSLA steel [4].

Solution of this problem in present work is based on a model of local non-equilibrium solidification (freezing) [5], which was supplemented by a model of solidification front interaction with the refractory in- 
oculants. The model taken as a basis is used for small as well as high rates of crystalline phase growth. Theoretical grounds of this model are made in work [6].

In two-dimensional medium the equations of model [5] describe a mass balance (1), evolution of solid and liquid phase interface and diffusion flow of additives $(2,3)$, growth of solid phase (4) and relationship of additive concentration in liquid and solid phases (5).

$$
\begin{gathered}
\frac{\partial}{\partial t}\left[(1-G)+C_{L}+k G C_{L}\right]+\frac{\partial J_{x}}{\partial x}+\frac{\partial J_{y}}{\partial y}=0, \\
\frac{\partial J_{x}}{\partial t}+J_{x}+\frac{1-G}{q} \frac{\partial C_{L}}{\partial x}=0, \\
\frac{\partial J_{y}}{\partial t}+J_{y}+\frac{1-G}{q} \frac{\partial C_{L}}{\partial y}=0, \\
\frac{\partial G}{\partial t}=\frac{\omega}{v} V, \\
C_{S}=k C_{L} .
\end{gathered}
$$

where $J_{x}$ and $J_{y}$ are the projections of vector $\mathbf{J}$ of diffusion flow on coordinate axis; $C_{L}$ and $C_{S}$ are the concentration in liquid and solid phases, respectively; $G$ is the fraction of solid phase in a cell [0-1]: $t$ is the time; $w$ is the area of phase interface in solidifying system; $v$ is the volume of two-phase area; $V$ is the rate of movement of intergrain boundary (interface of solid and liquid phases) along vector normal directed to liquid phase side; $k$ is the coefficient of additive non-equilibrium distribution.

It is known that small freezing rate and low overcooling promote for disturbance of microscopically smooth phase interface, it becomes morphologically unstable according to scenario of authors of work [7]. This is a classical example of development of cell-dendrite structure type, which is regulated by diffusion mass transfer in the locally equilibrium conditions. Increase of overcooling can result in deviation of local thermal-dynamic equilibrium in the system as well as initiation of quick freezing [8].

\section{Main assumptions of the used model:}

1. Isothermal two-phase system with constant pressure is considered.

2. The system consists of chemically inert binary-alloy, which solidifies from overcooled liquid.

3. Freezing from liquid phase can be so fast that rate $V$ of phase interface movement is compared on value with rate $V_{D}$ of additive diffusion.

4. Convection in liquid phase and diffusion in solid solidification phase are neglected.

5. Refractory inoculants are present in the liquid phase volume.

6. It is accepted that the refractory inoculants are uniformly distributed in the weld pool volume with some probability $\varphi(0 \leq \varphi \leq 1)$.
7. Refractory inoculants are stationary in solidification. All inoculants have similar size, comparable with size of cell of computational network $(\approx 0.4 \mu \mathrm{m})$.

Under these assumptions the process of dendrite structure development is regulated by mass transfer and kinetics of phase boundary movement. In particular, from assumption 4 it is necessary to take into account a finiteness of rate of diffusion transfer of additive and deviation from local equilibrium at phase boundary [9]. Thus, the model, describing the system, considers a local non-equilibrium diffusion of additive and kinetics of movement of phase interface.

Model of metal solidification process. A calculation area (solidification zone) is initiated at the beginning of calculation. It consists of solid phase of specific morphology (it can be separate cybotactic groupings randomly distributed in liquid phase, single nuclei of solid phase, plane front with inhomogeneities or without them, or their combinations) and liquid phase with determined initial additive concentration. A level of cell solidification is determined by fraction of solid phase in cell $G$. Each cell of the system can be liquid $(G=0)$ or completely solidified $(G=1)$, or belong to solidification front $(0<G<1)$, i.e. freeze in a present moment of time (Figure 1).

In order to determine an increment of fraction of solid phase $G$ it is necessary to calculate rate $V$ of phase interface. This should be done with the help of non-linear kinetic equation «rate-overcooling»

$$
V=\beta\left(\Delta T-\Delta T_{C}(V)-\Delta T_{N}(V)-\Delta T_{G},\right.
$$

where $\Delta T$ is the complete initial overcooling in the system; $\Delta T_{C}$ is the concentration overcooling caused by additive diffusion; $\Delta T_{N}$ is the overcooling caused by change of inclination of kinetic liquidus and determined by difference between equilibrium line of liquidus $T_{A}+m_{e} C_{0}$ and kinetic liquidus $T_{A}+m_{C} 0 ; \Delta T_{\mathrm{G}}$ is the overcooling caused by curvature of phase interface (Gibbs-Thompson effect).

Contributions of overcoolings are determined in the equations:

$$
\begin{gathered}
\Delta T=T_{A}+m_{e} C_{0}-T_{0}, \\
\Delta T_{C}=m(V)\left(C_{0}-C_{L}\right), \\
\Delta T_{N}=\left(m_{e}-m(V)\right) C_{0}, \\
\Delta T_{\mathrm{G}}=-\mathrm{G}\left(1-15 \cos \left(4\left(\theta_{1}-\theta_{0}\right)\right)\right) K .
\end{gathered}
$$

In the equations (6)-(10): $\beta$ is the kinetic coefficient of crystal growth; $T_{A}$ is the solidification (freezing) temperature of system main component; $T_{0}$ is the temperature in isothermal system; $m_{e}$ is the tangent of inclination angle of liquidus equilibrium line; $C_{0}$ is the initial concentration of additive in the melt; $m(V)$ is the inclination of kinetic liquidus; $G$ is the capillarity constant; $K$ is the curvature of phase interface; $\theta_{1}$ is the angle of orientation of vector-normal to phase 
interface; $\theta_{0}$ is the angle of orientation of the main crystallographic direction [10].

Capillarity constant $\mathrm{G}$ is determined as

$$
\mathrm{G}=\frac{\gamma}{\Delta S}, \quad \Delta S=\frac{Q_{L}}{T_{A}},
$$

where $\gamma$ is the surface tension; $\Delta S$ is change of entropy in solidification; $Q_{L}$ is the solidification latent heat.

In metal solidification process the weld pool is always characterized with presence of some stochastic process at phase interface caused by probabilistic processes. In order to get the results comparable with the experimental data, let's enter a kinetic coefficient of crystal growth in form of [11]

$$
\beta=1+\delta \eta(t, x, y)
$$

where $\delta \geq 0$ is the amplitude of stochastic noise; $\eta(t$, $x, y)$ is the probabilistic function taking the values in $[-1-1]$ interval for different points in $(t, x, y)$-space.

Serious effect on the results of computations of dendrites growth has artificial anisotropy, developed by computational mesh geometry [12]. In order to reduce this effect the computation of surface curvature considers an effect of not only the closest cells, but more distant neighbors. This work accounts effect of the cells being in a radius of three cells (Figure 2) with some weight coefficient. The weight coefficient depends on cell area, which is covered by radius circumference $3.5 h$ ( $h$ is the size of one cell in the computational mesh), the center of which is in the center of cell being computed. Thus, the computation of surface curvature in each separate cell takes into account effect of 44 neighbor points. A matrix of the weight coefficients $M$ is calculated one time and appears to be constant for all cells of the system. Numbers, shown in Figure, these are the values of weight coefficients of cell-neighbors.

Let's introduce a solidification vector $\bar{F}_{S}[10]$ for formalizing a computation of surface curvature. A value of this vector corresponds to sum of solid phase in radius circumference $3.5 \mathrm{~h}$ around the cell considering weight coefficients of matrix $M$, determined above.

$$
\left|\overrightarrow{F_{S}}\right|=\sum_{i, j=-3}^{3} G_{i, j} M_{i, j} \cdot
$$

Direction of vector corresponds to a line joining the mass center of all solid phase and center of studied cell. The coordinates of mass center relatively to studied cell in the Cartesian coordinates can be easily calculated on formulae:

$$
\Delta x=\sum_{i, j=-3}^{3} i G_{i, j} M_{i, j}, \Delta y=\sum_{i, j=-3}^{3} i G_{i, j} M_{i, j} .
$$

A vector of normal line of phase interface can be determined by formula

$$
\vec{n}=\frac{\overrightarrow{F_{S}}}{\left|\overrightarrow{F_{S}}\right|} .
$$

Thus, angle of orientation of vector-normal to phase interface $\theta_{1}$ in formula (10) is computed by formula

$$
\theta_{I}=\arctan \left(\frac{\Delta x}{\Delta y}\right) .
$$

Curvature of the surface in studied cell containing some fraction of solid phase $G$ is computed following from semiempirical dependence:

$$
K=\frac{A+B G-\left|\vec{F}_{S}\right|}{S} \frac{2}{h},
$$

where

$$
\begin{gathered}
S=\sum_{i, j=-3}^{3} M_{i, j}=38.484, \quad A=\sum_{i=-3}^{-1} \sum_{j=-3}^{3} M_{i, j}=15.754, \\
B=\sum_{j=-3}^{3} M_{0, j}=6.976,
\end{gathered}
$$

where $S, A, B$ are the constants determined from the matrix of weight coefficients; $h$ is the size of cell in the computational mesh.

Geometrically these constants are determined as different sections of the circle of $3.5 \mathrm{~h}$ radius (Figure 3); $S$ is the area of whole circle $(S=2 A+$ $\left.B=3.5^{2} \pi\right) ; A$ is the area of sector «cde», and $B$ is the area of domain «abcd». It is easy to see that equation (16) takes the zero value of surface curvature coefficient $K$ independent on solid phase fraction $G$ in the studied cell at plane solidification front. When modulus of vector $\bar{F}_{S}$ is less than $A+B G$ sum, then the surface is convex, in the opposite case it is concave, that can be seen in Figure 3.

Formula (16) is the modification of a method of surface curvature determination proposed in work [13]. This method imposes limitations on size of the cells and radius of matrix of the weight coefficients, which should be sufficiently small in comparison with typical scales of modeled structure.

For computation of fraction of additive, which is pushed out by newly-formed solid phase into the melt, let's determine a coefficient of non-equilibrium distribution of additive, which depends on local solidification rate and being calculated using equation

$$
k(V)=\left\{\begin{array}{l}
\frac{\left(1-V^{2}\right) k_{e}+V r}{1-V^{2}+V r}, \quad V<1, \quad r=\frac{V_{D}}{V_{D I}}, \\
1, V \geq 1,
\end{array}\right.
$$

where $k_{e}$ is the equilibrium coefficient of distribution; $V_{D}$ is the rate of additive diffusion in a liquid phase volume; $V_{D l}$ is the rate of additive diffusion at phase interface.

Inclination $m(V)$ of the kinetic liquidus for calculation of overcooling $\Delta T_{N}$ and $\Delta T_{C}$ is determined by 
coefficient of non-equilibrium distribution $k(V)(17)$ and rate $V$

$$
m(V)=\frac{m_{e}\left(1-k+\ln \left(\frac{k}{k_{e}}\right)+(1-k)^{2} V\right)}{1-k_{e}} .
$$

Updating of a concentration field takes place for each cell of computational domain following from mass balance and diffusion flow of additive. Concentration of $C_{S}$ additive in completely solid cells $(G=1)$ remains the same.

There is a surge of concentration at a diffusion wave front $\langle D F\rangle$ (leading edge of diffusion profile which moves with rate $V_{D}$ ) [5] in process of local non-equilibrium transfer of additive in the liquid phase volume. Detailed analysis of this effect can be found in work [11]. The boundary conditions at the diffusion wave front are set as boundary conditions of the first order and are as follows

$$
J_{D F}^{-}=\exp \left(-\left(\frac{\xi}{4} \tau\right)^{1 / 2}\right)\left(C_{F}-C_{0}\right) \mathbf{n},
$$

where $J_{D F}^{-}$is the outgoing flow of additive; $C_{F}-C_{0}$ is the concentration surge at a diffusion front; $\mathbf{n}$ is the vector of normal of diffusion front; $\xi$ is the parameter of stability of calculation scheme; $\tau$ is the time step.

Effect of the refractory inoculants on solidification process. Analysis of series of experimental data [2, 4] allowed taking for computations a hypothesis assuming that the solidification front absorbs the inoculant and surface tension in zone of contact is changed at the moment of contact.

In order to consider the effect of refractory inoculants on solidification front movement it is necessary to compute a value of interphase tension between the inoculant and melt $\left(\sigma_{12}\right)$ using table data [14].

$$
\sigma_{12}=\sigma_{1}+\sigma_{2}-W_{a}
$$

where $\sigma_{12}$ is the value of interphase tension between refractory inoculant and melt; $\sigma_{1}$ is the inoculant surface tension; $\sigma_{2}$ is the alloy surface tension; $W_{a}$ is the adhesion work.

Surface tension of the melt is computed based on procedure proposed by S. Popel [15]:

$$
\sigma_{2}=\sigma_{\mathrm{Fe}}-2000 \lg \Sigma F_{i} X_{i},
$$

where $\sigma_{\mathrm{Fe}}$ is the surface tension of pure iron; $F_{i}$ is the parameter characterizing capillary activity of alloying additive; $x_{i}$ is the atomic fraction of $i^{\text {th }}$ alloy component.

The boundary conditions are not set in common understanding (Figure 4). Initially there are completely solid cells in the bottom of computational domain, computation in which is not carried out. From the right and from the left the computational domain forms a «cylinder» by means of development of virtual data, to which data from the cells being closest to the boundary of computational domain are copied. It is done for emulation of position of computational domain in the weld pool, where development of the primary structure in it can also be effected by growth of dendrites in metal adjacent volumes. From above the computational area is limited by a layer of cells, computation in which is not carried out. This develops the layer, which does not prevent propagation of diffusion wave that physically corresponds to weld pool large dimensions.

The model described above made a basis for development of a program for tracing the movement of phase interface in solidification of weld metal of HSLA steels in time depending on initial conditions (initial solidification rate, initial morphology of solid phase, inclination angle of a vector of the most intensive heat rejection to solidification surface) and physical parameters of alloy (solidification temperature of alloy main component, growth kinetic coefficient, tangent of inclination angle of liquidus equilibrium line, equilibrium coefficient of additive distribution, coefficient of additive diffusion, solidification latent heat, rate of additive diffusion in volume and on phase interface, stochastic noise amplitude).

Based on developed software a series of computational experiments was carried out, the results of which are given below.

Evaluation of melt composition effect on solidification process. Composition of weld metal of HSLA steel (wt.\%): $0.049 \mathrm{C} ; 0.298 \mathrm{Si} ; 1.39 \mathrm{Mn}$; $0.023 \mathrm{~S} ; 0.015 \mathrm{P} ; 0.15 \mathrm{Cr} ; 2.26 \mathrm{Ni} ; 0.25 \mathrm{Mo} ; 0.039 \mathrm{Al}$; $0.008 \mathrm{Ti}$ was taken as a basis for computational experiments. Initial rate of solidification was accepted $0.27 V_{D}$. Physical parameters of alloy are given in the Table. Figure 5 shows difference in size and morphology of dendrites, which were developed for similar period of time, without considering the effect of alloying elements on alloy surface tension (Figure 5, a) and taking into account the effect alloying elements (Figure 5, b).

Results of computational experiments of effect of refractory inoculants introduction on dendrite metal structure. Titanium dioxide $\left(\mathrm{TiO}_{2}\right)$ was taken as introduced inoculant for the computations. It has the following parameters of surface interaction with iron melt, namely melting temperature $\left(T_{m}\right) 1834{ }^{\circ} \mathrm{C}$; surface tension of liquid phase $\left(\sigma_{1}\right) 1780 \mathrm{~mJ} / \mathrm{m}^{2}$; limiting wetting angle $(\theta) 0$ degree; adhesion work $\left(W_{a}\right)$ $3560 \mathrm{~mJ} / \mathrm{m}^{2}$. A parameter of refractory inoculants distribution in the weld pool metal $\varphi$ was taken equal 0 , $0.1,0.2$ and 0.3 . The results of computations showing evolution of the dendrite structure in time with different density of inoculants distribution in the weld pool metal are given in Figures 6-9, respectively. 


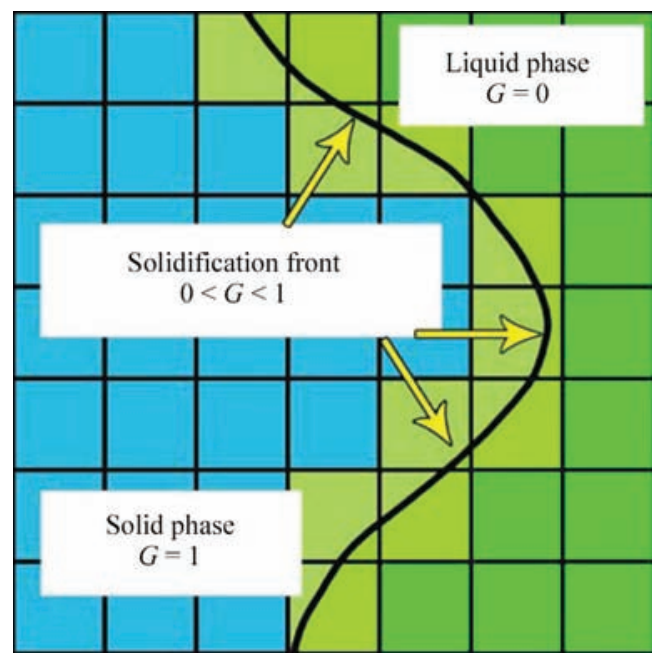

Figure 1. Value of fraction of solid phase $\mathrm{G}$ for liquid and solid phases and solidification front

Thus, a branched solidification structure with wide branches of dendrites (up to $100 \mu \mathrm{m}$ ) can be observed in absence (Figure 6) of the refractory inoculants in the weld pool.

The weld pool feeding with the refractory inoculants, affecting the surface tension between solid phase and melt, promoted for a change of dendrite structure from branched one to completely columnar morphology (Figures 7-9). Such a significant change of nature of the weld metal primary structure is only caused by the processes taking place on a contact surface of refractory inoculant with growing dendrite.

In this case $\mathrm{TiO}_{2}$ is well wetted by iron melt that promoted for increase of local solidification rate in a contact zone of growing dendrite with inoculant. This occurs due to rise of overcooling value in the contact zone (Gibbs-Thompson, $\Delta T_{\mathrm{G}}$ effect). Increase of rate of dendrite growth due to Gibbs-Thomson effect results in rise of concentration overcooling $\left(\Delta T_{C}\right)$ due to pushing out a large amount of additive before the

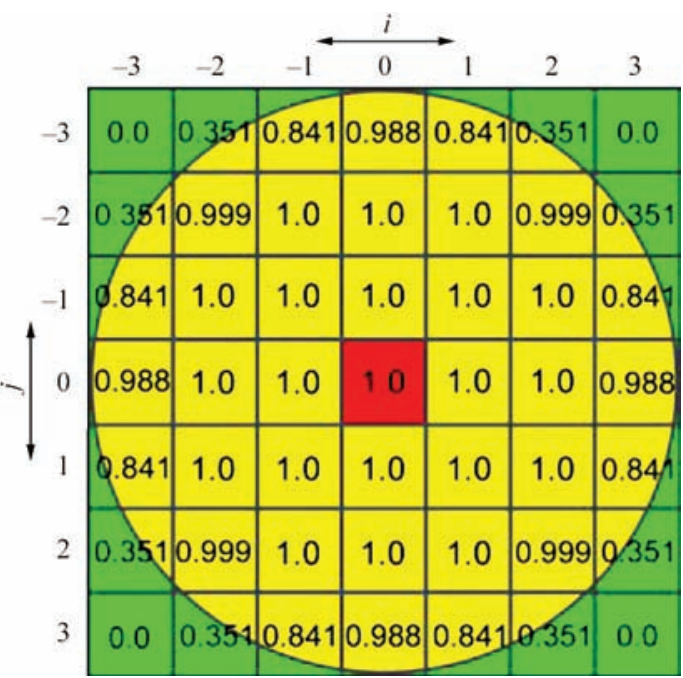

Figure 2. Matrix of weight coefficients for determination of surface curvature [10]

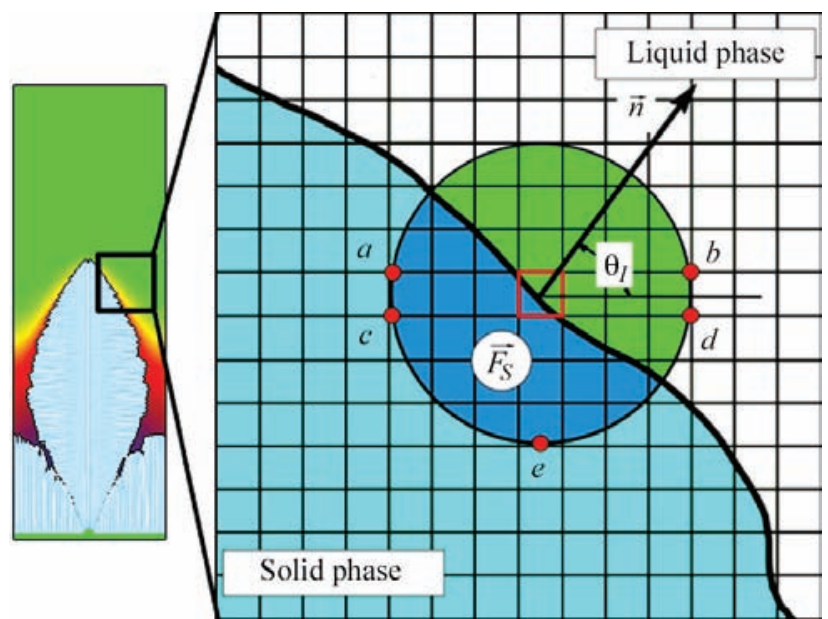

Figure 3. Determination of solidification vector and computation of surface curvature [16]

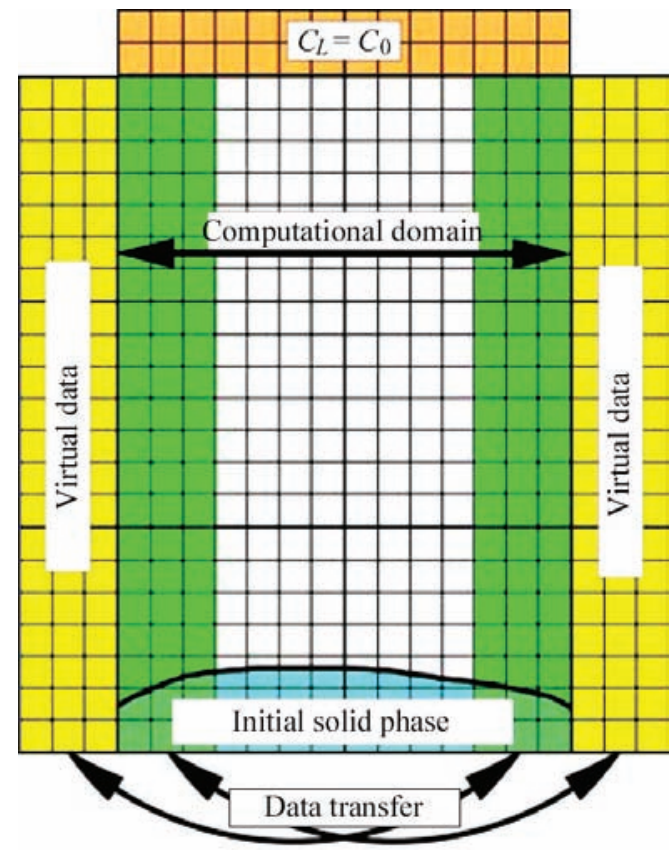

Figure 4. Rules at computational domain boundaries [10]

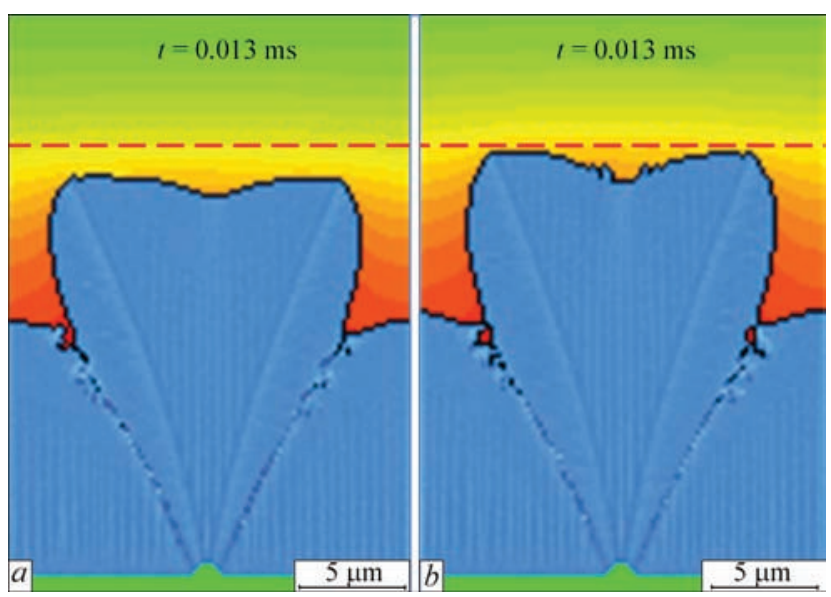

Figure 5. Results of computations of solidification start: $a$ without effect of alloying elements; $b$ - with alloying elements effect 


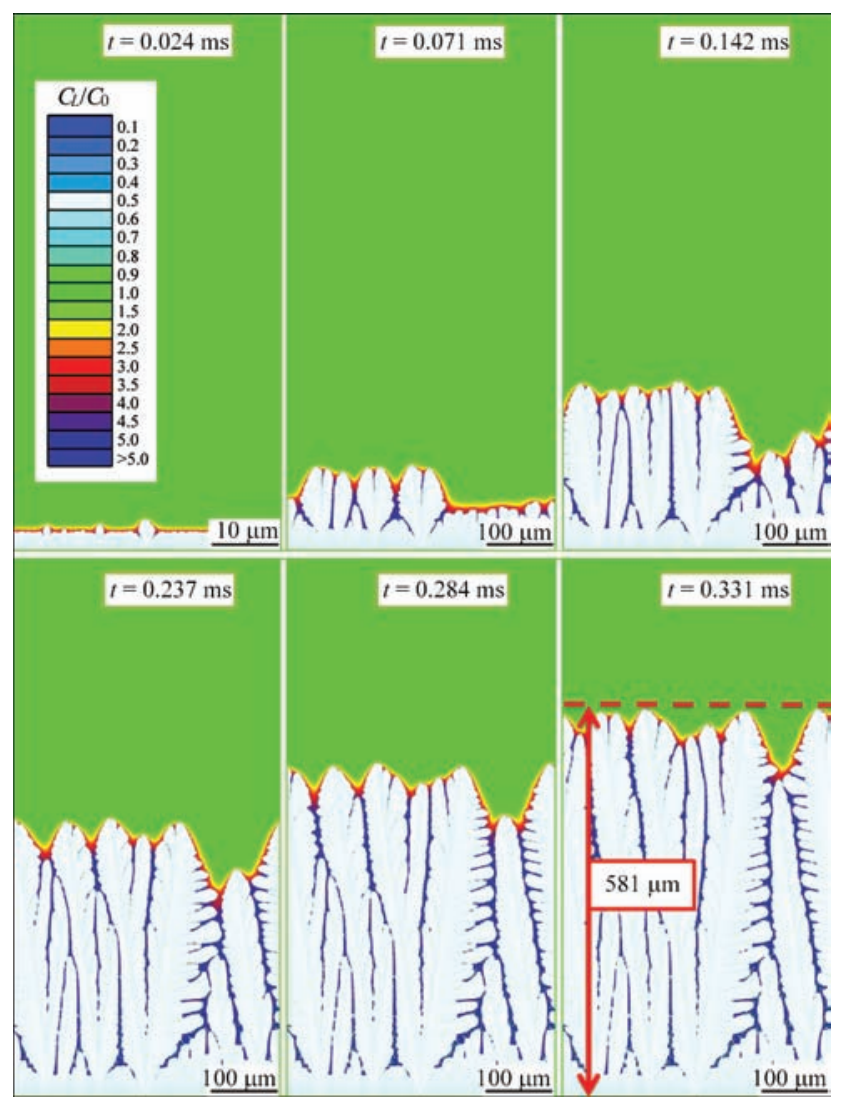

Figure 6. Evolution of dendrite structure in time considering refractory inoculants $(\varphi=0)$

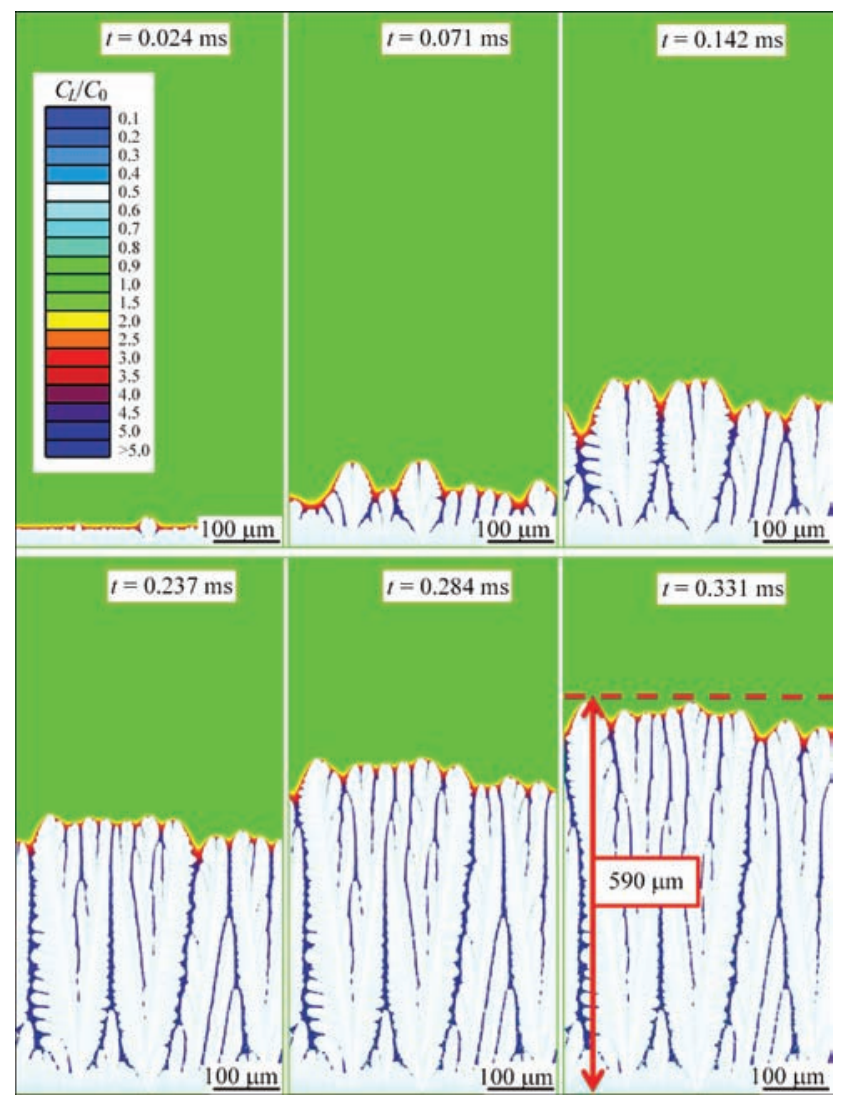

Figure 7. Evolution of dendrite structure in time considering refractory inoculants $(\varphi=0.1)$ 
Values of physical parameters taken in computation

\begin{tabular}{|l|c|c|c|}
\hline \multicolumn{1}{|c|}{ Physical parameters } & Designations & Unit of measurement & Value \\
\hline Temperature of main component freezing & $T_{A}$ & $\mathrm{~K}$ & 1809 \\
\hline Kinetic coefficient of growth & $\beta$ & $\mathrm{m} /(\mathrm{s} \cdot \mathrm{K})$ & 0.4 \\
\hline Tangent of inclination angle of liquidus equilibrium line & $m_{e}$ & $\mathrm{~K} / \mathrm{wt} . \%$ & -80 \\
\hline Distribution equilibrium coefficient & $k_{e}$ & - & 0.1 \\
\hline Coefficient of additive diffusion & $D$ & $\mathrm{~m}^{2} / \mathrm{s}$ & $6 \cdot 10^{-8}$ \\
\hline Solidification latent heat & $Q_{L}$ & $\mathrm{~J} / \mathrm{m}^{3}$ & $10^{9}$ \\
\hline Diffusion rate in volume & $V_{D}$ & $\mathrm{~m} / \mathrm{s}$ & 17 \\
\hline Diffusion rate at phase interface & $V_{D l}$ & $\mathrm{~m} / \mathrm{s}$ & 17 \\
\hline Amplitude of stochastic noise & $\delta$ & - & 0.07 \\
\hline
\end{tabular}
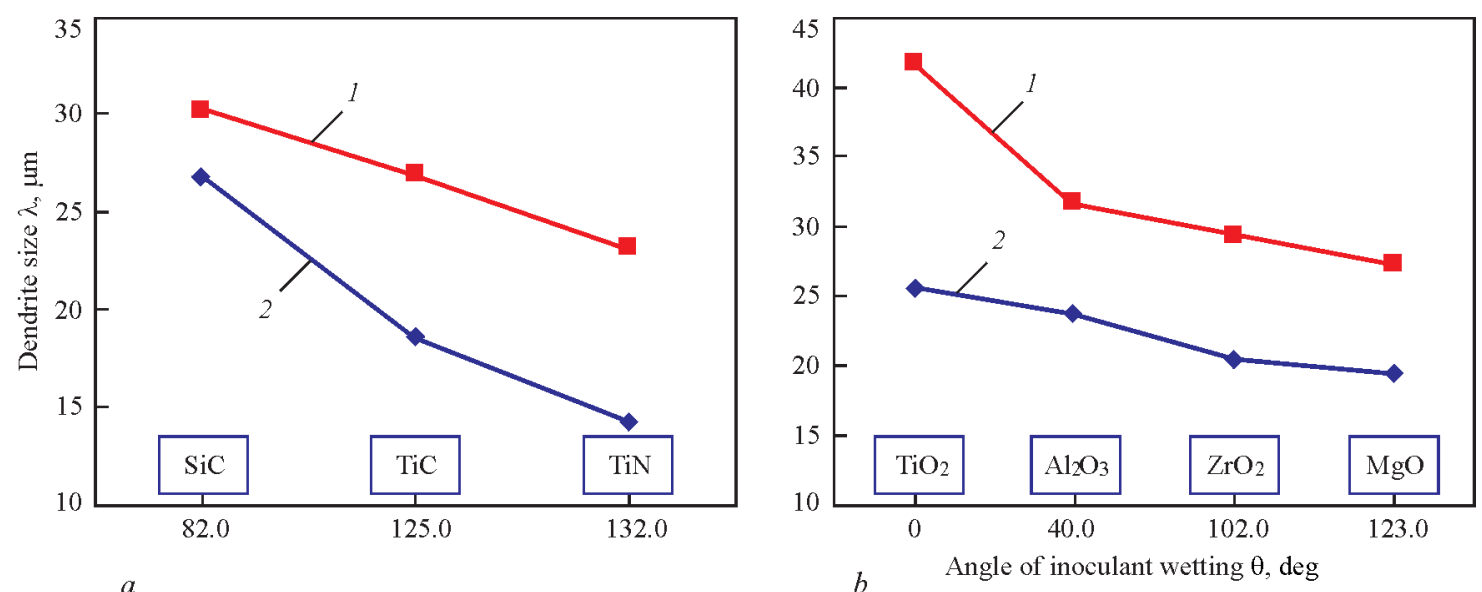

Figure 10. Comparison of experimental results of measurement and computational data of parameters of metal primary structure of specimens in the first $(a)$ and second $(b)$ series of experiments: $1-\lambda_{\text {lexp }} ; 2-\lambda_{\text {1comp }}$

solidification front. Also, change of growth rate at the end of dendrite promotes for further deviation of the process from equilibrium and rise of overcooling due to deviation from liquidus equilibrium line $\left(\Delta T_{N}\right)$.

Hypothetical description of nature of development of the physical processes, imbedded in the proposed solidification model, allows simulating quality changes of the weld metal dendrite structure depending on number and properties of introduced inoculants. If refractory inoculants are absent, the solidification front in the weld pool moves for $581 \mu \mathrm{m}$ during 0.331 ms (Figure 6), and at their uniform distribution with $\varphi=0.3$ probability this makes $802 \mu \mathrm{m}$ (Figure 9) all other things being equal (including initial solidification rate). It is necessary to note significant change of morphology of the dendrite structure from much branched, formed because of competitive growth of separate crystals, to completely columnar dendrites. Reduction of wetting angle in the local points of contact of growing dendrite with metal melt can provide significant qualitative change of the dendrite structure, that, in turn effect further solid phase transformations in metal and as a result final microstructure and mechanical properties of HSLA steel weld metal.

Figure $10 a, b$ presents a comparison of dimensions of the dendrite primary structure, received in computational and experimental way $[2,17]$ for the specimens of the first and second series, respectively. An average error of data, obtained by means of computational experiment, makes around $25 \%$ and increases with rise of wetting angle of the refractory inoculant by weld pool metal. Such a difference in received results can be related with selection of parameters of distribution of the refractory inoculants in the weld pool metal $\varphi$ equal 0.3 . It apparently does not correspond to the conditions of carried experiment researches. This factor should be taken into account in further investigations and it is necessary to study in more details the effect of distribution parameter on development of the weld metal primary structure. Also, the error was introduced by inaccurate composition and model limitations.

\section{Conclusions}

The model, based on finite element method coupled with cellular automation method, well suits simulation of solidification process of weld pool metal taking into account the effect of the refractory inoculants. It is related with the fact that the main model of crystalline structure development can be easily completed by additional rules and laws of interaction of moving 
solidification front with inclusions introduced in the weld metal volume. Also, an advantage of such approach is the possibility of tracing of any parameters of cellular automation discrete cells (concentration of additive, overcooling value, coefficient of surface curvature etc.) in time.

Computational experiments, carried with the help of developed software, showed the possibility of regulation of parameters and morphology of metal primary structure by means of melt feeding with the refractory disperse inoculants. Developed mathematical model and software, written on its basis, are good for prediction of dimensional parameters and morphology of weld metal primary structure considering refractory inoculants introduced in the weld pool.

1. Sandeep, J., Chhibber, R., Mehta, N.P. (2012) Issues in welding of HSLA steels. Advanced Materials Research, 365, 4449.

2. Golovko, V.V., Pokhodnya, I.K. (2013) Effect of non-metallic inclusions on formation of structure of the weld metal in highstrength low-alloy steels. The Paton Welding J., 6, 2-10.

3. Ermolenko, D.Yu., Golovko, V.V. (2014) Numerical modeling and prediction of weld microstructure in high-strength steel welding (Review). Ibid., 3, 2-10.

4. Golovko, V.V., Stepanyuk, S.N., Ermolenko, D.Yu. (2015) Effect of titanium-containing inoculants on structure and properties of weld metal of high-strength low-alloy steels. Ibid., 2, 14-18.

5. Galenko, P.K., Krivilev, M.D. (2000) Isothermal growth of crystals in undercooled binary alloys. Matematicheskoe Modelirovanie, 12(11), 17-37.

6. Galenko, P., Danilov, D. (1997) Local nonequilibrium effect on rapid dendritic growth in binary alloy melt. Physics Letters A, 235(3), 271-280.
7. Mullins, W.W., Sekerka, R.F. (1964) Stability of the planar interface during crystallization of a dilute binary alloy. J. Applied Physics, 35, 444-459.

8. Kurz, W., Fisher, D.J. (1992) Fundamentals of solidification. Transact. Tech. Publications Ltd.

9. Galenko,P., Sobolev, S. (1997) Local nonequilibrium effect on undercooling in rapid solidification of alloys. Physical Review E, 55(1), 343-352.

10. Pavlyk, V. (2004) Modeling and direct numerical simulation of dendritic structures under solidification conditions during fusion welding. Aachen: Shaker Verlag GmbH.

11. Galenko, P.K., Krivilev, M.D. (2000) Finite-difference scheme for modeling of crystalline structure formation in undercooled binary alloys. Matematicheskoe Modelirovanie, 12(12), 11-13.

12. Guillemot, G., Gandin, C.-A., Combeau, H. (2006) Modeling of macrosegregation and solidification grain structures with a coupled cellular automaton-finite element model. ISIJ Int., 46(6), 880-895.

13. Sasikumar, R., Sreenivasan, R. (1994) 2-dimensional simulation of dendrite morphology. Acta Metallurgica et Materialia, 7(2), 2381-2386.

14. Panasyuk, A.D., Fomenko, V.S., Glebova, G.G. (1986) Resistance of non-metallic materials in melts. Kiev: Naukova Dumka.

15. Popel, S.I. (1971) Theory of metallurgical processes. Moscow: VINITI.

16. Diltey, U., Pavlik, V., Raihel, T. (1997) Computer modeling of formation of weld metal microstructure in fusion welding. Avtomatich. Svarka, 3, 3-9.

17. Golovko, V.V., Stepanyuk, S.N., Ermolenko, D.Yu. (2014) Technology of welding of high-strength low-alloy steels by introduction of titanium-containing inoculants. In: Nanodimensional Systems and Nanomaterials: Research in Ukraine. Monography. Ed. by A.G. Naumovets. Kiev: Akademperiodika. 